\title{
ONE-DIMENSIONAL PATTERN FORMATION IN A MODEL OF BURNING
}

\author{
LAWRENCE K. FORBES ${ }^{1}$
}

(Received 12 November 1991; revised 24 February 1992)

\begin{abstract}
We discuss a model of a burning process, essentially due to Sal'nikov, in which a substrate undergoes a two-stage decay through some intermediate chemical to form a final product. The second stage of the process occurs at a temperature-sensitive rate, and is also responsible for the production of heat. The effects of thermal conduction are included, and the intermediate chemical is assumed to be capable of diffusion through the decomposing substrate. The governing equations thus form a reaction-diffusion system, and spatially inhomogeneous behaviour is therefore possible.

This paper is concerned with stationary patterns of temperature and chemical concentration in the model. A numerical method for the solution of the goveming equations is outlined, and makes use of a Fourier-series representation of the pattern. The question of the stability of these patterns is discussed in detail, and a linearised solution is presented, which is valid for patterns of very small amplitude. The results of accurate solutions to the fully non-linear equations are discussed, and compared with the predictions of the linearised theory. Parameter regions in which there exists genuine nonuniqueness of solutions are identified.
\end{abstract}

\section{Introduction}

The formation of patterns in reaction-diffusion systems is now a much studied subject, and there are applications in a remarkably wide variety of areas. Turing [17] explained morphogenesis in embryos in terms of a biochemical reaction-diffusion system, in which a spatially-homogeneous steady state would become unstable, giving rise instead to a pattern of chemical concentration

\footnotetext{
'Dept of Mathematics, University of Queensland, St. Lucia, Queensland, 4072, Australia. (C) Australian Mathematical Society, 1993, Serial-fee code 0334-2700/93
} 
upon the embryo surface. This general behaviour type is now known as a "Turing bifurcation", and has been invoked more recently by Murray [13], for example, to account for the regular patterns of stripes and spots on animal coats. Pattern formation has also been observed in aggregations of single-celled organisms, and the phenomenon has been described using a reaction-diffusion system of equations by Tyson, Alexander, Manoranjan and Murray [19]. Various inorganic chemical reactions are now known to admit patterns, such as the celebrated Belousov-Zhabotinskir reaction (see Tyson [18] and Winfree [21]). The formation of patterns in combustion processes has been described by Brindley, Jivraj, Merkin and Scott [2] and by Gray and Scott [9] (chapter 10), and reaction-diffusion systems of Ginzburg-Landau type are currently receiving much attention as a means of explaining spiral defects and other non-linear optical phenomena (see Rica and Tirapegui [14]). Recently, McNabb and Bass [12] have used non-linear reaction-diffusion equations to model the uptake of protein-bound ligands by biological cells.

In the present paper, we examine the formation of patterns in a reactiondiffusion system which arises from a simple model of a burning process. The model was apparently first proposed by Sal'nikov [15], and is adapted slightly here to allow for spatial inhomogeneities. The burning process is imagined to be occurring at the surface of some substrate $S$, which decomposes in a sequence of two first-order reactions, through some intermediate chemical $C$ to form a final product $P$. The reaction scheme is simply

$$
S \stackrel{k_{0}}{\rightarrow} C \stackrel{k_{1}}{\rightarrow} P
$$

The concentration [S] of substrate $S$ is taken to be a constant, invoking an assumption which is sometimes known as the "pool-chemical" approximation. In the present problem, this assumption might be regarded as corresponding to one of two physical situations. In the first, substrate $S$ could be continuously fed up to the reaction zone; in this case, the substrate might be a gaseous hydrocarbon, and the patterns would correspond to spatially inhomogeneous temperature distributions across the nozzle of an oxy-welding torch, for example. The experimental work of Griffiths [10] shows that the simple Sal'nikov reaction scheme (1.1) does indeed have some relevance to the combustion of acetaldehyde in oxygen. The second physical interpretation of the assumption of constant substrate concentration $[S]$ is one in which $S$ is a solid. The reaction zone at the surface of $S$ would then move through the solid substrate, and the inert product $P$ (soot) would be removed, perhaps by the action of wind. This 
interpretation thus corresponds to a situation in which there is uniform ablation of the substrate as a result of an internal chemical reaction.

If the intermediate chemical $C$ in the Sal'nikov scheme (1.1) is able to diffuse through the decomposing substrate $S$, then the kinetic rate equation for this intermediate species is

$$
\frac{\partial[C]}{\partial t}=D \nabla^{2}[C]+k_{0}[S]-k_{1}[C],
$$

where $D$ is the diffusion coefficient for quantity $C$. In the Sal'nikov scheme (1.1), the first reaction is assumed to take place isothermally, with constant rate $k_{0}$, but the second stage produces heat, and occurs at a temperature-dependent rate $k_{1}$ given by the Arrhenius law

$$
k_{1}(T)=M_{1} \exp (-E /(R T)) .
$$

Here, $M_{1}$ is a rate constant having units seconds ${ }^{-1}, E$ is the activation energy for the reaction, $R$ is the universal gas constant, and $T$ denotes absolute temperature.

In view of the involvement of the temperature $T$ in the above rate equation, an energy-balance equation is also required. Suppose that substrate $S$ occupies some planar region $\Omega$, with boundary $\partial \Omega$, and let $\mathcal{R}$ be an arbitrary region within $\Omega$, having boundary $\partial \mathcal{R}$. The conservation of energy within region $\mathcal{R}$ per unit width in the reaction zone then leads to the equation

$$
\frac{d}{d t} \iint_{\mathcal{R}} \rho c_{h} T d A=-\oint_{\partial \mathcal{R}} \mathbf{q} \cdot \mathbf{n} d \ell+\iint_{\mathcal{R}} Q d A,
$$

in which $\rho$ is the density of the medium and $c_{h}$ is its heat capacity. The symbol $q$ denotes a heat flux vector per unit width at the boundary $\partial \mathcal{R}$ of the arbitrary region, which has normal $\mathbf{n}$ directed out of the region $\mathcal{R}$. The quantity $Q$ represents the sum of the energy per unit time per unit surface area which is generated internally by the second exothermic stage of the scheme (1.1) and that which is gained or lost through the surface.

According to Fourier's law of conduction, the heat-flux vector $\mathbf{q}$ is given by

$$
\mathbf{q}=-K \nabla T,
$$

where $K$ is a heat conduction coefficient. The quantity $Q$ is comprised of a loss term due to Newtonian cooling at the surface, in a direction perpendicular to the planar region $\Omega$, as indicated in Figure 1. There is also a term representing the internal heat production, and hence

$$
Q=-\chi\left(T-T_{a}\right)+Q_{1} k_{1}[C]
$$




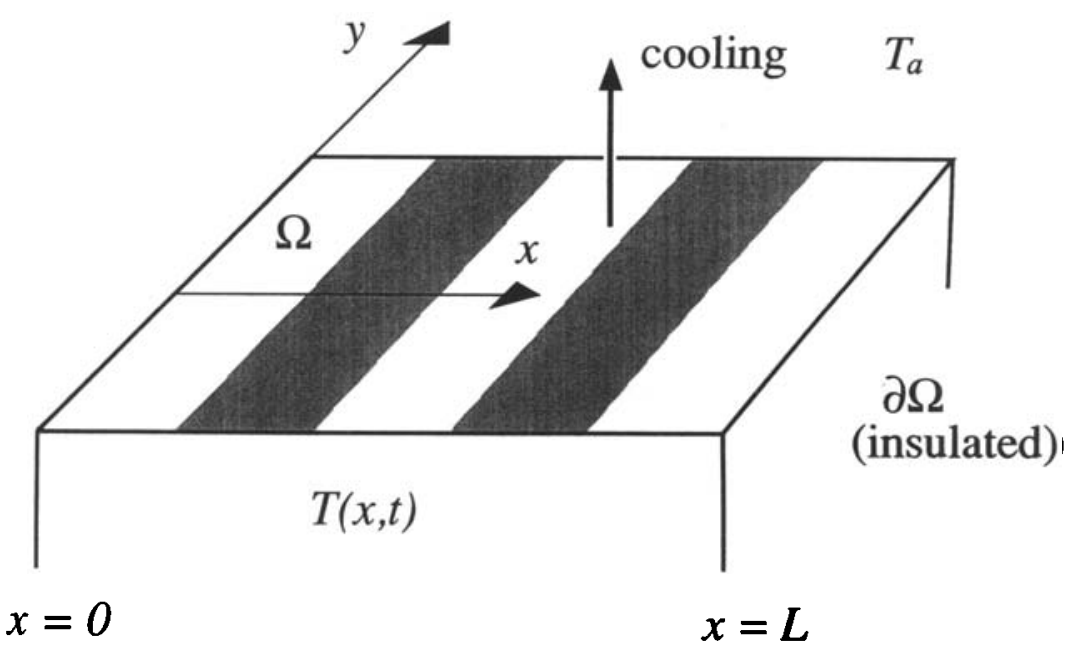

FIGURE 1. Sketch of the physical model, showing the burning face $\Omega$ and its (insulated) boundary $\partial \Omega$. The surface pattern is one-dimensional, and is indicated by the shaded regions shown.

in which $\chi$ and $Q_{1}$ are proportionality constants for the Newtonian cooling term and the heat production term, respectively. The ambient temperature is $T_{a}$.

Expressions (1.5) and (1.6) are substituted into the conservation relation (1.4), and Green's theorem in the plane is used to convert the single integral term to an integral over the planar region $\mathcal{R}$. In view of the arbitrariness of this region within $\Omega$, we therefore obtain the conservation law in differential form

$$
\rho c_{h} \frac{\partial T}{\partial t}=K \nabla^{2} T-\chi\left(T-T_{a}\right)+Q_{1} k_{1}[C]
$$

It is convenient now to introduce dimensionless variables, by scaling the temperature with respect to the reference quantity $E / R$ and the concentration with reference to $\left(\rho c_{h} E\right)\left(Q_{1} R\right)^{-1}$. Time is made dimensionless with respect to $1 / M_{1}$, and all lengths are referred to $L$, which is some measure of the size of the domain $\Omega$. It may then be seen that solutions to the present problem are dependent upon the five dimensionless parameter groups

$$
\begin{gathered}
\sigma=\frac{D}{M_{1} L^{2}}, \quad \mu=\frac{k_{0}[S] Q_{1} R}{M_{1} \rho c_{h} E}, \quad \alpha=\frac{K}{M_{1} \rho c_{h} L^{2}}, \\
\quad \beta=\frac{\chi}{M_{1} \rho c_{h}}, \quad \theta_{a}=\frac{R T_{a}}{E} .
\end{gathered}
$$


The first of these represents the dimensionless diffusion coefficient of the intermediate chemical $C$, and the second is effectively a measure of the substrate concentration. The constant $\alpha$ corresponds to the thermal conductivity of the medium, $\beta$ is the coefficient of Newtonian cooling through the substrate surface, and the parameter $\theta_{a}$ is a measure of the ambient temperature. In these dimensionless variables, the governing reaction-diffusion equations (1.2), (1.3) and (1.7) become

$$
\begin{aligned}
& \frac{\partial C}{\partial t}=\sigma \nabla^{2} C+\mu-C e^{-1 / T}, \\
& \frac{\partial T}{\partial t}=\alpha \nabla^{2} T-\beta\left(T-\theta_{a}\right)+C e^{-1 / T} \quad \text { within } \Omega .
\end{aligned}
$$

We assume that the boundary of the region is perfectly insulated, and is impervious to any of the reacting species. The boundary conditions appropriate to this problem are therefore the Neumann conditions

$$
\frac{\partial C}{\partial n}=\frac{\partial T}{\partial n}=0 \quad \text { on } \partial \Omega .
$$

In the original model of Sal'nikov [15], the effects of chemical diffusion and thermal conductivity were not included, and so spatial inhomogeneity was not a possibility. This corresponds to a situation in which the reagents are perfectly well mixed in the present model, and is equivalent to setting our parameters $\sigma$ and $\alpha$ to zero. Equations (1.8) then become a system of two ordinary differential equations for the evolution of the concentration $C$ and temperature $T$ in time, and Sal'nikov [15] showed that limit cycles were possible, corresponding to periodic oscillations in the concentration and temperature.

Chemical oscillations are now well documented in isothermal reactions (e.g. Tyson [18]), and have been observed for temperature dependent reactions similar to the present Sal'nikov model by Griffiths [10]. He noted two types of oscillatory behaviour, a cool-flame oscillation which occurred almost at uniform temperature, and a more violent type, which involved large temperature variations and was accompanied by flashes of light. The Sal'nikov model ((1.8) with $\sigma=\alpha=0$ ) has since been studied by Gray, Kay and Scott [8], using the Frank-Kamenetskii approximation to the full Arrhenius exponential term in (1.3). They identified the Hopf bifurcation points, at which the homogeneous steady state becomes unstable, and a stable oscillatory solution emerges instead.

In a later paper, Kay and Scott [11] analysed the exact equations with the full exponential non-linearity, and showed that there are parameter regions in which 
two oscillatory solutions can exist simultaneously; one of these is stable and the other unstable. This situation comes about as a result of a subcritical Hopf bifurcation occurring at one of the two possible Hopf points. Gray and Roberts [7] have also examined the fully non-linear Sal'nikov model, and applied degenerate singularity theory in an attempt to build up a global description of the solution behaviour. Forbes [4] has recently established rigorously the parameter values for which oscillatory behaviour is not possible, and has introduced a shooting method for computing periodic solutions when they occur. This approach automatically determined whether or not the limit cycle was stable.

When chemical diffusion and heat conduction are permitted ( $\sigma$ and $\alpha$ are non-zero), oscillatory behaviour is now not limited to temporal variations only, but can also occur spatially. The time-dependent kinematic waves of chemical activity so produced may be structures of great topological complexity, such as the "spiral" and "scroll" waves described by Winfree [21] and Winfree and Jahnke [22] in the case of the isothermal Belousov-Zhabotinskii equation, and by Winfree [20] in more general excitable media.

Genuine pattern formation occurs as a limiting case of these spatio-temporal waves, when the propagation speed is zero. A time-independent structure is then observed. In this paper, we compute such patterns in the Sal'nikov model (1.8), allowing variation in a single space dimension only. The patterns would thus appear as a series of stripes across the face of the burning material, as in Figure 1. The Neumann boundary conditions (1.9) ensure that this is a nonlinear eigenvalue problem, and so the pattern amplitude is usually required to be specified in advance, and one of the physical parameters is to be determined as part of the solution. A numerical method for solving the one-dimensional problem is outlined in Section 2, and is similar to that used by Forbes [5], [6], since the concentration $C(x)$ and temperature $T(x)$ are represented in terms of Fourier series, with coefficients that are to be found numerically.

Becker and Field [1] computed stationary patterns in the Belousov-Zhabotinskiı̌ reaction using a method which integrated the governing reaction-diffusion equations forward in time until some stationary state was reached. This approach guaranteed that the pattern thus obtained would be stable. However, Forbes [5] obtained patterns directly as solutions to the steady equations, and no such guarantee of stability is available to this solution technique. Indeed, Comstock and Field [3] have shown that Forbes' solutions are unstable, with the result that they probably would not be observable in the laboratory. The question of stability is thus a very significant concern for solution methods like those of the present paper, and was addressed in a later paper by Forbes [6] for the 
Belousov-Zhabotinskiǔ reaction. He succeeded in producing patterns which were quasi-stable, with a life-time sufficiently long to be observable.

In the present paper, we treat the problem of pattern stability in some detail, since the solution technique we employ is capable of computing stable and unstable patterns with equal ease. In Section 3, an eigenvalue approach is used to determine whether any Fourier component of an arbitrary applied disturbance is capable of growing without bound, so rendering the pattern unstable. We identify the regions of parameter space within which the stable and unstable solution types may be produced.

A linearised theory, valid for patterns of very small amplitude, is presented in Section 4, and the stability of the linearised patterns is related to the onset of oscillatory behaviour in the well-stirred Sal'nikov model. In Section 5, we present the results of extensive numerical experimentation, and Section 6 contains some concluding remarks.

\section{The spectral solution technique}

In this section, we present a numerical method for the solution of the onedimensional steady equations which govern the form of the concentration and temperature profiles $C(x)$ and $T(x)$, respectively. Equations (1.8) thus become

$$
\begin{aligned}
\sigma C^{\prime \prime}(x)+\mu-C(x) e^{-1 / T(x)} & =0, \\
\alpha T^{\prime \prime}(x)-\beta\left(T(x)-\theta_{a}\right)+C(x) e^{-1 / T(x)} & =0 \quad \text { in } \quad 0 \leq x \leq 1,
\end{aligned}
$$

with boundary conditions

$$
C^{\prime}(x)=T^{\prime}(x)=0 \quad \text { on } \quad x=0,1 .
$$

Consideration of (2.1) with their associated boundary conditions (2.2) shows this system to constitute a non-linear eigenvalue problem. It therefore follows that a sequence of eigenmodes is to be expected. In addition, there will be some degree of arbitrariness in the pattern amplitude, but one of the physical parameters will need to be computed as the eigenvalue.

Following Forbes [5], [6], the $m$-th eigenfunction is therefore sought in the Fourier-series form

$$
C(x)=C_{0}+\sum_{p=1}^{\infty} C_{p} \cos (m p \pi x)
$$




$$
T(x)=T_{0}+\sum_{p=1}^{\infty} T_{p} \cos (m p \pi x) .
$$

This form (2.3) automatically satisfies the Neumann boundary conditions (2.2), but the coefficients $C_{0}, C_{1}, C_{2}, \ldots$ and $T_{0}, T_{1}, T_{2}, \ldots$ are as yet unknown. In addition, we shall compute the chemical diffusion coefficient $\sigma$ as the non-linear eigenvalue of the problem. The arbitrariness associated with the eigenfunction (2.3) is removed by specifying the concentration amplitude

$$
A_{C}=C(0)-C(1 / m),
$$

which, at least for the linearised solution, represents the difference between the maximum and minimum points in the concentration profile, occurring at the points $x=0$ and $x=1 / m$ for the $m$-th eigensolution.

The numerical solution technique begins with the observation that, when the two equations in the system (2.1) are added, the resulting equation relating $C$ and $T$ and their derivatives is linear. Consequently, it is possible to express all the Fourier coefficients $T_{0}, T_{1}, T_{2}, \ldots$ simply in terms of $C_{0}, C_{1}, C_{2}, \ldots$ The relationship is

$$
\begin{aligned}
& T_{0}=\theta_{a}+\mu / \beta, \\
& T_{p}=-\frac{\sigma(m p \pi)^{2} C_{p}}{\alpha(m p \pi)^{2}+\beta}, \quad p=1,2, \ldots
\end{aligned}
$$

The infinite Fourier series (2.3) are next truncated, and approximated by trigonometric polynomials of some order $P$. It is therefore required to find the $(P+1)$-vector of unknowns

$$
\mathbf{u}=\left[C_{0}, \sigma, C_{2}, \ldots, C_{P}\right]^{\top} .
$$

An initial guess is made for the vector (2.6), and is updated iteratively using Newton's method. On the basis of the current estimate for the vector (2.6) of unknowns, all other quantities of interest can be computed. The coefficient $C_{1}$ is obtained from the amplitude condition (2.4), and is thus given by the expression

$$
C_{1}=\frac{1}{2} A_{C}-\sum_{r=1}^{[(P-1) / 2]} C_{2 r+1} .
$$

The square brackets around an expression indicates that only the integer part of that quantity is to be taken. The Fourier coefficients for the temperature profile are finally obtained from (2.5), up to the required order $P$. 
It remains to satisfy the first of the equations in the system (2.1), and this is done by adjusting the elements of the vector (2.6) iteratively, using Newton's method. The equation is multiplied successively by the basis functions 1 , $\cos (m \pi x), \ldots, \cos (m P \pi x)$ and integrated over the interval $0 \leq x \leq 1$ to give the system of non-linear algebraic equations

$$
\begin{aligned}
& E_{0}=\mu-\int_{0}^{1} C e^{-1 / T} d x=0, \\
& E_{p}=-\frac{1}{2} \sigma(m p \pi)^{2} C_{p}-\int_{0}^{1} C e^{-1 / T} \cos (m p \pi x) d x=0 p=1,2, \ldots, P
\end{aligned}
$$

The integrals in these expressions are evaluated using the composite trapezoidal rule, with values for the concentration $C(x)$ and temperature $T(x)$ taken from the (truncated) Fourier expansions (2.3), evaluated at the equally-spaced mesh points

$$
x_{j}=(j-1) /(J-1), \quad j=1,2, \ldots, J .
$$

Since the integrands are periodic, trapezoidal-rule quadrature is of exponentially high order accuracy.

The algebraic problem is thus to force the vector $\left[E_{0}, E_{1}, \ldots, E_{P}\right]^{\top}$ of residuals in (2.8) to be within some pre-set distance of the zero vector. "This is achieved using a damped Newton's method, as in Forbes [6].

\section{Stability of the nonlinear steady patterns}

Suppose that a steady pattern of chemical concentration and temperature has been found, by the methods of Section 2 . In this section, we denote such a steady pattern as $\left[C_{s}(x), T_{s}(x)\right]^{\top}$, and seek to determine whether it is stable to small perturbations. Since stability here means that a small disturbance will not grow with time, it is necessary to consider the full time-dependent equations (1.8). A small time-dependent perturbation to the steady solution is now assumed, in the form

$$
\begin{aligned}
& C(x, t)=C_{s}(x)+\epsilon C_{1}(x, t)+\mathrm{O}\left(\epsilon^{2}\right), \\
& T(x, t)=T_{s}(x)+\epsilon T_{1}(x, t)+\mathrm{O}\left(\epsilon^{2}\right),
\end{aligned}
$$

where $\epsilon$ is some small parameter. Equations (3.1) are now substituted into the full equations (1.8) and boundary conditions (1.9), and only terms of first order 
in $\epsilon$ are retained. This gives rise to the first-order variational equations

$$
\begin{aligned}
& \frac{\partial \mathbf{U}_{1}}{\partial t}=\mathbf{D}_{m} \frac{\partial^{2} \mathbf{U}_{1}}{\partial x^{2}}+\mathbf{A}_{m}(x) \mathbf{U}_{1} \quad \text { in } \quad 0 \leq x \leq 1, \\
& \frac{\partial \mathbf{U}_{1}}{\partial x}=\mathbf{0} \quad \text { on } \quad x=0,1 .
\end{aligned}
$$

Here, we have defined the vector

$$
\mathrm{U}_{1}(x, t)=\left[C_{1}(x, t), T_{1}(x, t)\right]^{\top},
$$

the diagonal matrix

$$
\mathbf{D}_{m}=\left[\begin{array}{ll}
\sigma & 0 \\
0 & \alpha
\end{array}\right]
$$

and the spatially dependent matrix

$$
\mathbf{A}_{m}(x)=\left[\begin{array}{cc}
-\exp \left(-1 / T_{s}\right) & -\left[C_{s} \exp \left(-1 / T_{s}\right)\right] / T_{s}^{2} \\
\exp \left(-1 / T_{s}\right) & -\beta+\left[C_{s} \exp \left(-1 / T_{s}\right)\right] / T_{s}^{2}
\end{array}\right] .
$$

The subscript $m$ has been employed with these two matrices, to signify that their elements are dependent upon the particular eigenfunction for which a steady solution has been found. The stability of a steady pattern to small perturbations may thus be decided by an investigation of solutions to the linear system of equations (3.2) for arbitrary initial conditions.

The general solution to the vector system of equations (3.2) clearly has the Fourier-series form

$$
\mathbf{U}_{1}(x, t)=\sum_{n=0}^{\infty} \mathbf{V}_{n}(t) \cos (n \pi x) .
$$

A steady pattern computed by the method of Section 2 will be stable to small perturbations if and only if the Fourier coefficients $V_{n}(t)$ cannot grow with the passage of time.

When (3.3) is substituted into the variational system (3.2) and the latter is then spectrally decomposed, there results an infinite vector differential equation for the evolution of the Fourier coefficients in (3.3). This equation is

$$
\begin{gathered}
\mathbf{V}_{0}^{\prime}(t)=\sum_{q=0}^{\infty}\left(\int_{0}^{1} \mathbf{A}_{m}(x) \cos (q \pi x) d x\right) \mathbf{V}_{q}(t) \\
\mathbf{V}_{n}^{\prime}(t)=-n^{2} \pi^{2} \mathbf{D}_{m} \mathbf{V}_{n}(t)+2 \sum_{q=0}^{\infty}\left(\int_{0}^{1} \mathbf{A}_{m}(x) \cos (q \pi x) \cos (n \pi x) d x\right) \mathbf{V}_{q}(t) \\
n=1,2,3 \ldots
\end{gathered}
$$


Progress in the study of (3.4) cannot proceed without some approximation, and so we truncate the Fourier series (3.3) beyond the $(N+1)$-th order term, for some integer $N$. We define the sequence of constant $2 \times 2$ matrices

$$
\begin{aligned}
\mathbf{S}_{q, n}=\int_{0}^{1} \mathbf{A}_{m}(x) \cos (q \pi x) \cos (n \pi x) d x, \\
q=0,1,2, \ldots \quad n=0,1,2, \ldots
\end{aligned}
$$

and consider the truncated approximation to the system (3.4) in the vector form

$$
\mathbf{X}^{\prime}(t)=\mathbf{H} \mathbf{X}(t)
$$

in which we have defined the $2 N+2$ partitioned vector

$$
\mathbf{X}(t)=\left[\mathbf{V}_{0}(t), \mathbf{V}_{1}(t), \ldots, \mathbf{V}_{N}(t)\right]^{\top}
$$

and the $(2 N+2) \times(2 N+2)$ constant partitioned matrix

$$
\mathbf{H}=\left[\begin{array}{cccc}
\mathbf{S}_{0,0} & \mathbf{S}_{1,0} & \cdots & \mathbf{S}_{N, 0} \\
2 \mathbf{S}_{0,1} & 2 \mathbf{S}_{1,1}-\pi^{2} \mathbf{D}_{m} & \cdots & 2 \mathbf{S}_{N, 1} \\
\vdots & \vdots & \ddots & \vdots \\
2 \mathbf{S}_{0, N} & 2 \mathbf{S}_{1, N} & \cdots & 2 \mathbf{S}_{N, N}-N^{2} \pi^{2} \mathbf{D}_{m}
\end{array}\right]
$$

The matrices (3.5) are evaluated with great accuracy using the trapezoidal rule, and values of the steady concentration and temperature taken from the Fourierseries representation (2.3).

For stability of the computed pattern (2.3), it is both necessary and sufficient that the eigenvalues of the matrix $\mathbf{H}$ all have negative real parts, in the limit $N \rightarrow$ $\infty$. We use the NAG library routine F02AFF to compute all the eigenvalues of the finite matrix $\mathbf{H}$ in (3.6), and stability of the corresponding pattern is then decided on this basis. Essentially the only error made in this process is the truncation of the infinite system (3.4) to the finite system (3.6). However, this is not a significant concern, since an examination of (3.4) shows that the higher-order Fourier modes are dominated by a structure of the form $\mathbf{V}_{n}{ }^{\prime}(t) \approx$ $-n^{2} \pi^{2} \mathbf{D}_{m} \mathbf{V}_{n}(t)$ for $n$ sufficiently large. Thus the high-order Fourier components decay rapidly to zero. A similar situation was found in the problem studied by Forbes [6]. In practice, we find that taking $N=30$ is easily adequate to account for the low-order Fourier modes. 


\section{The linearised solution}

In this section, we investigate patterns of very small amplitude. In practice, these are possibly not of great interest, since they would be too small to be detected in a laboratory experiment. Nevertheless, this investigation is of value mathematically, since it allows simple closed-form expressions to be obtained for the pattern profile and for the eigenvalue $\sigma$, and additionally permits a rather complete analysis of pattern stability, which serves as a useful guide to the fully non-linear numerical approach.

The linearised equations are derived from the exact non-linear system (1.8) by means of the perturbation expansions

$$
\begin{aligned}
& C(x, t)=C_{e}+\epsilon C_{1}(x, t)+\mathrm{O}\left(\epsilon^{2}\right), \\
& T(x, t)=T_{e}+\epsilon T_{1}(x, t)+\mathrm{O}\left(\epsilon^{2}\right),
\end{aligned}
$$

where $\epsilon$ is some small parameter related to the pattern amplitude, as in Section 3. When (4.1) are substituted into (1.8) and only terms of zeroth order in the parameter $\epsilon$ are retained, we obtain the equilibrium concentration and temperature

$$
\begin{aligned}
C_{e} & =\mu \exp \left\{1 /\left(\theta_{a}+\mu / \beta\right)\right\}, \\
T_{e} & =\theta_{a}+\mu / \beta .
\end{aligned}
$$

The first-order terms in the expansion (4.1) give rise to the linearised equations

$$
\begin{aligned}
& \frac{\partial \mathbf{U}}{\partial t}=\mathbf{D}_{m} \frac{\partial^{2} \mathbf{U}}{\partial x^{2}}+\mathbf{A U} \quad \text { in } \quad 0 \leq x \leq 1, \\
& \frac{\partial \mathbf{U}}{\partial x}=\mathbf{0} . \quad \text { on } \quad x=0,1,
\end{aligned}
$$

where the vector $\mathbf{U}$ contains the first-order perturbation functions in the form

$$
\mathbf{U}(x, t)=\left[C_{1}(x, t), T_{1}(x, t)\right]^{\top} .
$$

The diagonal matrix $\mathbf{D}_{m}$ is exactly as defined in the variational system of equations (3.2) and contains the eigenvalue $\sigma$ (the chemical diffusion constant), and the constant matrix $\mathbf{A}$ is

$$
\mathbf{A}=\left[\begin{array}{cc}
-\exp \left(-1 / T_{e}\right) & -\mu / T_{e}^{2} \\
\exp \left(-1 / T_{e}\right) & -\beta+\mu / T_{e}^{2}
\end{array}\right]
$$


A stationary pattern is a steady solution to the system (4.3), and can be written

$$
\mathbf{U}(x)=\mathbf{B} \cos (m \pi x),
$$

where $\mathbf{B}$ is an arbitrary constant vector. The integer $m$ is free to be chosen, and as in (2.3), it indicates which eigenfunction is to be sought. When (4.4) is substituted into the linearised equations (4.3), there results the eigenvalue problem

$$
\mathbf{A B}=m^{2} \pi^{2} \mathbf{D}_{m} \mathbf{B} .
$$

From (4.5), the vector $B$ may be found, within an arbitrary constant of multiplication which might then be decided by an amplitude condition such as (2.4). In addition, the diffusion coefficient $\sigma$ is determined as the eigenvalue of the system (4.5), and has the form

$$
\sigma=\frac{T_{e}^{2}\left(\alpha m^{2} \pi^{2}+\beta\right) \exp \left(-1 / T_{e}\right)}{m^{2} \pi^{2}\left[\mu-T_{e}^{2}\left(\alpha m^{2} \pi^{2}+\beta\right)\right]},
$$

in which the constant $T_{e}$ is the equilibrium steady-state temperature defined in (4.2).

The formula (4.6) for the diffusion coefficient $\sigma$ imposes restrictions on the region in parameter space within which patterns (of infinitesimal amplitude) are possible. The results are only of physical significance if $\sigma$ is a non-negative constant, which therefore bounds the thermal diffusion coefficient $\alpha$ according to the formula

$$
\alpha<\frac{1}{m^{2} \pi^{2}}\left[\frac{\mu}{T_{e}^{2}}-\beta\right]
$$

Since the coefficient of thermal diffusion itself cannot be negative in any application of physical interest, there thus arises from (4.7) a further restriction

$$
\theta_{a}<\sqrt{\mu / \beta}-\mu / \beta
$$

which shows that pattern formation is only possible within this bounded interval of ambient temperatures $\theta_{a}$. Finally, the positivity of ambient temperature in (4.8) gives the condition $\mu<\beta$. From this, we see that pattern formation can only occur if the ambient temperature is not too hot, and if Newtonian cooling of the burning surface occurs at a sufficiently great rate.

It is now instructive to consider the stability of these linearised patterns (4.4). The general solution to (4.3) has the Fourier-series form

$$
\mathbf{U}(x, t)=\sum_{q=0}^{\infty} \cos (q \pi x) \sum_{r=1}^{2} \exp \left(\lambda_{q, r} t\right) K_{q, r}
$$


The exponents $\lambda_{q, r}$ and the vectors $\mathbf{K}_{q, r}$ are eigenvalues and eigenvectors of the matrix equations

$$
\left[\mathbf{A}-q^{2} \pi^{2} \mathbf{D}_{m}\right] \mathbf{K}_{q, r}=\lambda_{q, r} \mathbf{K}_{q, r}, \quad q=0,1,2, \ldots
$$

The linearised steady pattern (4.4) can only be stable if all the constants $\lambda_{q, r}$ have negative real parts, given that one of these constants is zero when $q=m$. (The equivalent condition for stability of small amplitude patterns in the BelousovZhabotinskiĭ reaction has been given by Forbes [6].) We define the trace and the determinant of the $2 \times 2$ matrices in (4.10) to be, respectively,

$$
\begin{aligned}
& \Sigma_{m, q}=-q^{2} \pi^{2}(\alpha+\sigma)-\left(\beta-\mu / T_{e}^{2}+e^{-1 / T_{e}}\right), \\
& \Delta_{m, q}=q^{4} \pi^{4} \alpha \sigma+q^{2} \pi^{2}\left[\alpha e^{-1 / T_{e}}+\sigma\left(\beta-\mu / T_{e}^{2}\right)\right]+\beta e^{-1 / T_{e}}
\end{aligned}
$$

and obtain the solution for the exponents $\lambda_{q, r}$ in the form

$$
\lambda_{q, r}=\frac{1}{2}\left[\Sigma_{m, q} \pm \sqrt{\Sigma_{m, q}^{2}-4 \Delta_{m, q}}\right] .
$$

The eigenvalues $\lambda_{q, r}$ will all have negative real parts if the trace $\Sigma_{m, q}$ in (4.11) is negative, and the determinant $\Delta_{m, q}$ is positive. Under these conditions, the linearised steady solution (4.4) will be stable.

From (4.11), a necessary condition for stability, as revealed by an investigation of the trace $\Sigma_{m, q}$, is that

$$
f\left(\theta_{a}, \mu ; \beta\right)=\beta-\mu / T_{e}^{2}+e^{-1 / T_{e}}>0 .
$$

This condition is related to the appearance of time-dependent oscillations in the well-stirred system (see Forbes [4] and Gray and Scott [9]). When the system is well mixed, there are no spatial gradients, and this is equivalent to setting the parameters $\alpha$ and $\sigma$ to zero in (1.8). This leaves a pair of coupled ordinary differential equations for the time evolution of the concentration $C$ and temperature $T$, and the condition $f\left(\theta_{a}, \mu ; \beta\right)=0$ indicates points of Hopf bifurcation, at which a time-oscillatory solution is born from the equilibrium conditions (4.2). The necessary condition (4.13) therefore states that linearised patterns can only be stable if they occur outside the region within which temporal oscillations exist in the well-stirred system.

Figure 2 shows the location of the Hopf line $f\left(\theta_{a}, \mu ; \beta\right)=0$ in the $\theta_{a}-\mu$ parameter plane, for $\beta=1$. The curve is qualitatively similar for other values 


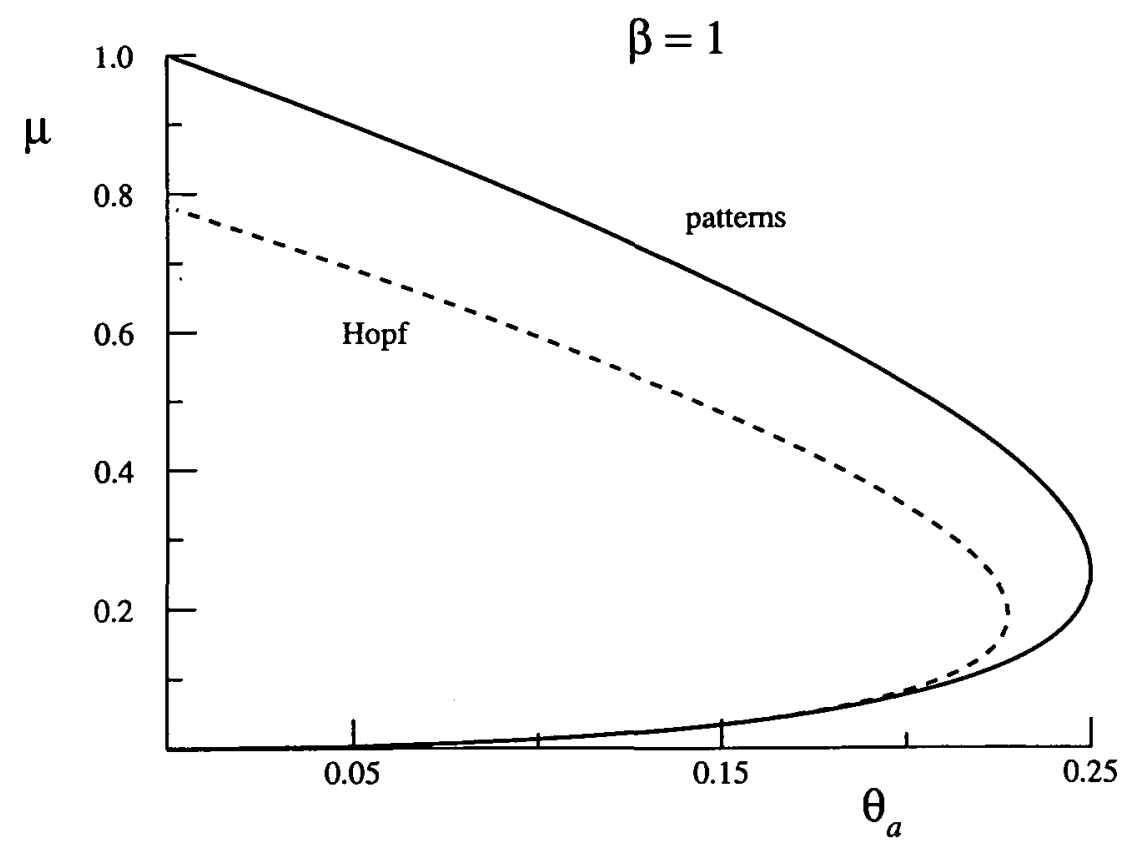

FIGURE 2. The location of the Hopf curve (dashed line) in the $\theta_{a}-\mu$ parameter plane, for Newtonian cooling coefficient $\beta=1$. Also shown is the border of the region (4.8) within which linearised patterns are possible (solid line).

of the Newtonian cooling coefficient $\beta$. Inequality (4.13) shows that stable linearised patterns are only possible above and to the right of the Hopf curve in Figure 2 (sketched with a dashed line). Patterns which are formed within the region enclosed by the Hopf curve are therefore unstable, and utimately decay to a well-mixed time-oscillatory solution of (1.8). Also shown on Figure 2 is the bounding curve for ambient temperature $\theta_{a}$, obtained from (4.8). Pattern formation is only possible in the region to the left of this (solid) curve.

A situation of some interest is the limiting case $\alpha=0$, when there is no thermal diffusion. Under such circumstances, stable patterns of infinitesimal amplitude are never possible, as the following theorem shows.

THEOREM 1. All time-independent small amplitude patterns (4.4) are unstable if $\alpha=0$.

PROOF. In the degenerate case $\alpha=0$, the determinant $\Delta_{m, q}$ in (4.11) becomes 
simply

$$
\Delta_{m, q}=q^{2} \pi^{2} \sigma\left(\beta-\mu / T_{e}^{2}\right)+\beta e^{-1 / T_{e}} .
$$

Now in view of the condition (4.8) for the existence of patterns of physical significance, this determinant can be made arbitrarily large and negative, by choosing to perturb the steady pattern (4.4) with a signal having sufficiently large wave number $q$. Therefore, exponents $\lambda_{q, r}$ exist which have positive real parts, by (4.12), and hence the pattern is unstable.

In discussing the stability of chemical patterns, it is usually the case that the lowest Fourier modes are the ones which are most likely to cause pattern instability (Forbes [6]). In Theorem 1, however, we see that the instability is caused by the high modes, which is an interesting reversal of the usual situation. When thermal diffusivity is present $(\alpha \neq 0)$, however, these high modes are rapidly damped out.

Figures 3(a) and (b) show the variation of the chemical diffusion coefficient $\sigma$ with ambient temperature $\theta_{a}$, for the cases $\alpha=0$ and $\alpha=0.001$, respectively. Here, we have taken $\mu=0.5$ and $\beta=1$, and the diffusion coefficient, computed from (4.6), is shown for the first four eigensolutions in each case. When $\alpha=0$, there is a positive value of $\sigma$ for each eigenfunction parameter $m$, for every ambient temperature $\theta_{a}$ permitted by (4.8). However, for non-zero $\alpha$ as in Figure 3(b), only a finite number of eigenfunctions give positive values for $\sigma$ at a given ambient temperature $\theta_{a}$, and there will therefore only be a finite spectrum of physically meaningful eigensolutions for any ambient temperature. We refer to these figures again in the following section.

\section{Nonlinear patterns}

Here, numerical results obtained by the method of Section 2 and the stability test in Section 3 are presented, analysed, and compared with the linearised results of Section 4.

We begin by considering the degenerate case $\alpha=0$, for which there is no thermal diffusion. The results of many numerical solutions are summarised in Figure 4, which shows the dependence of the non-linear eigenvalue $\sigma$ (the chemical diffusion coefficient) upon the pattern amplitude as defined by (2.4). Here we have taken $\theta_{a}=0.18, \mu=0.5$ and $\beta=1$, which represents a point between the two curves in Figure 2, where patterns are normally expected to exist, and to be stable. However, by Theorem 1, small amplitude patterns will in fact be unstable in this singular case $\alpha=0$. The stability test of Section 3 
(a)

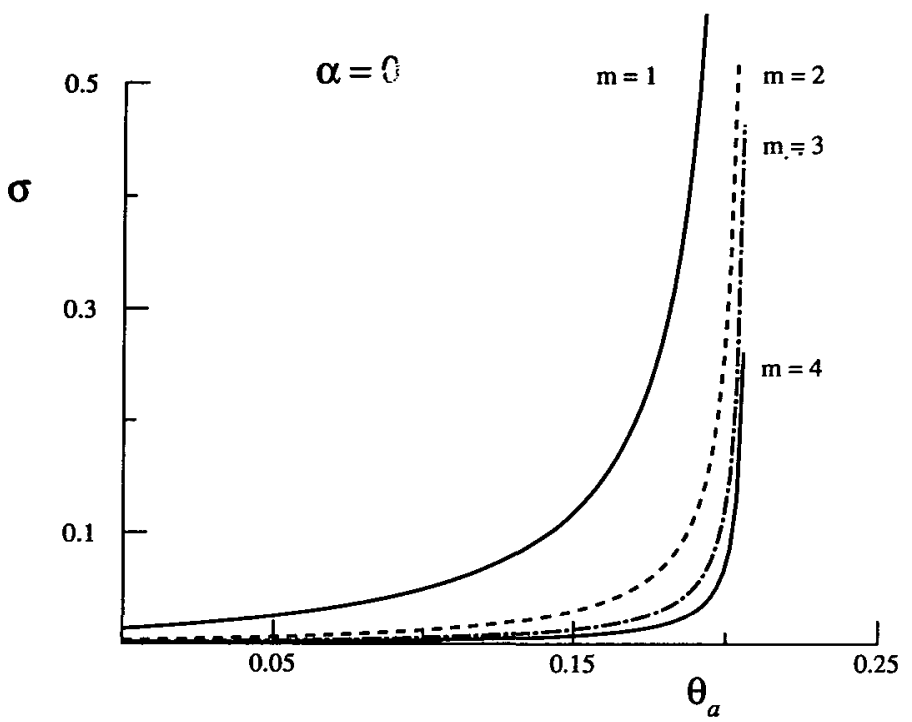

(b)

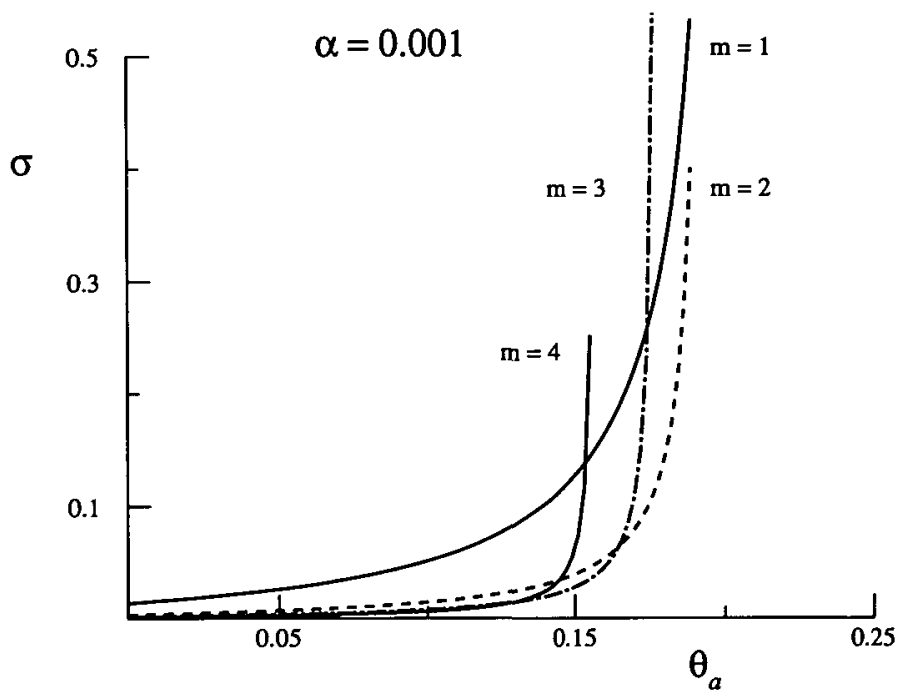

FIGURE 3. The variation of the diffusion coefficient $\sigma$ with ambient temperature $\theta_{a}$, for the linearised solution. Results are shown for the first four eigensolutions $m=1,2,3,4$, assuming parameter values $\mu=0.5$ and $\beta=1$. (a) For $\alpha=0$, (b) For $\alpha=0.001$. 


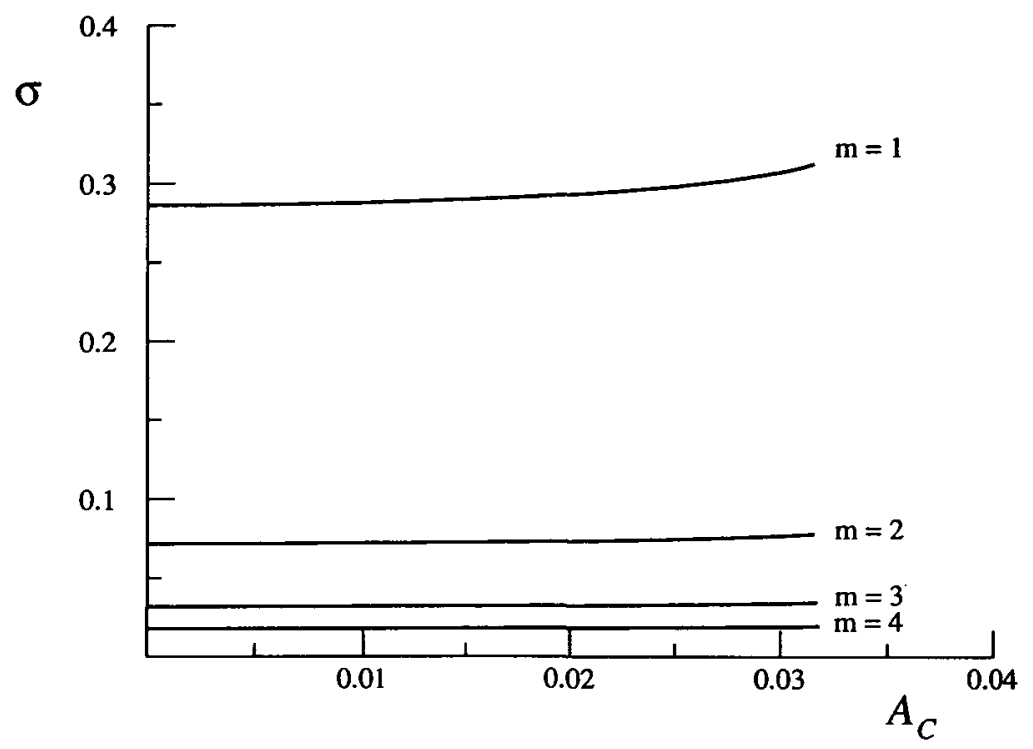

FIGURE 4. The variation of the diffusion coefficient $\sigma$ with pattern amplitude $A_{C}$, for $\theta_{a}=0.18$, $\mu=0.5, \beta=1$ and $\alpha=0$. Results are shown for the first four eigensolutions $m=1,2,3,4$.

confirms that the prediction of Theorem 1 also has validity in the non-linear case, since all the solutions in Figure 4 are unstable. Results are shown for the first four eigenfunctions, $m=1,2,3,4$.

As $A_{C} \rightarrow 0$, the results agree very well with those of the linearised solution (4.6). There is then surprisingly little change in the computed values of $\sigma$ as the pattern amplitude $A_{C}$ is increased. However, the non-linear solution branches all fail suddenly at about $A_{C}=0.0315$, and this intriguing limiting behaviour is now analysed.

Temperature profiles are shown in Figures 5(a) and 5(b), for the first eigenfunction $m=1$ and the fourth eigenfunction $m=4$, respectively. In both graphs, we have taken $\theta_{a}=0.18, \mu=0.5, \beta=1$ and $\alpha=0$, and in each case we have plotted a pattern of moderate amplitude $A_{C}=0.015$ (shown with a dashed line), and the pattern of the largest amplitude that could be computed with our numerical technique (sketched with a solid line). There is, in fact, no qualitative difference between the results in Figures 5(a) and 5(b), apart from a change in the length scale of the pattern. This is because, in the degenerate case $\alpha=0$, the governing equations (2.1) and (2.2) for steady profiles are invariant under the transformation $\tilde{x}=x / m, \tilde{\sigma}=\sigma / m^{2}$. Thus the pattern with $m=4$ 
(a)
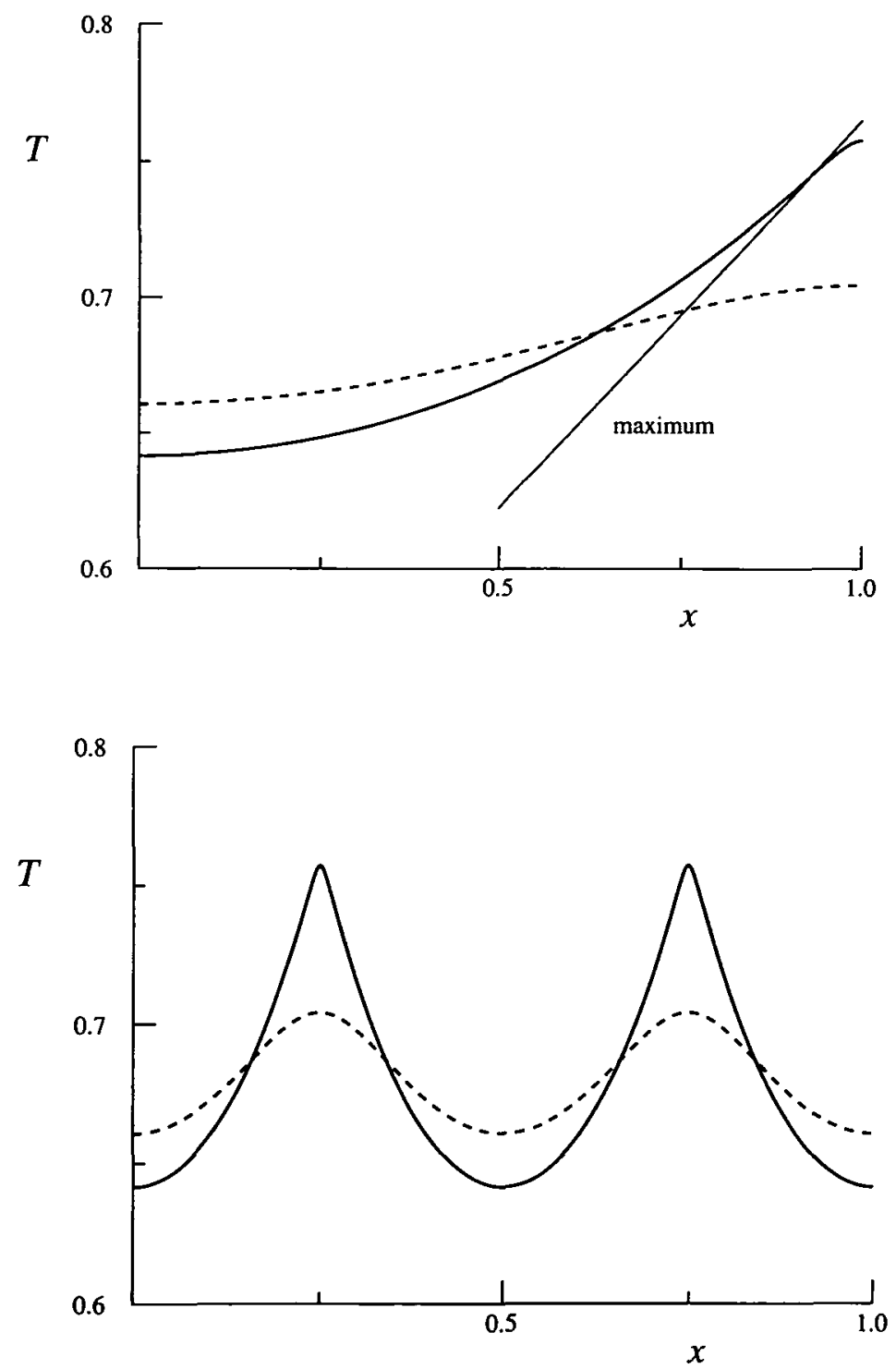

(b)

FIGURE 5. Unstable temperature profiles for $\theta_{a}=0.18, \mu=0.5, \beta=1$ and $\alpha=0$. Results are shown for (a) The first eigensolution $m=1$, with $A_{C}=0.015$ (dashed line) and $\sigma=0.312$ (solid line, $A_{C}=0.031533185$ ). The line labelled "maximum" is that predicted by Theorem 2 . (b) The fourth eigensolution $m=4$, with $A_{C}=0.015$ (dashed line) and $\sigma=0.0195$ (solid line, $A_{C}=0.031533185$ ) 
in Figure 5(b) is identical to the pattern in Figure 5(a) with $m=1$, except that its length scale has been compressed by a factor of four, and its eigenvalue $\sigma=0.0195$ is one-sixteenth of the value 0.312 for the limiting profile in Figure 5(a).

Figures 5 suggest a possible reason for the sudden failure of the numerical method to continue beyond about $A_{C}=0.0315$ in Figure 4 . It appears that the temperature profile of maximum amplitude is ultimately limited by the formation of a sharp corner at the crest, reminiscent of the famous Stokes gravity wave (see Schwartz and Fenton [16]). This is indeed the case, and is examined in the following theorem.

THEOREM 2. When thermal diffusion is absent, $\alpha=0$, the temperature profile of maximum amplitude has a corner at the maximum value of temperature

$$
T_{\max }=\left(1+\sqrt{1-4 \theta_{a}}\right) / 2,
$$

which encloses an angle $\pi-2 \arctan \gamma$, where

$$
\gamma=\left[\frac{T_{\max }^{2}\left[\mu-\beta\left(T_{\max }-\theta_{a}\right)\right] \exp \left(-1 / T_{\max }\right)}{\sigma \beta\left(1-2 T_{\max }\right)}\right]^{1 / 2} .
$$

PROOF. When $\alpha=0$, the steady concentration $C(x)$ may be eliminated from (2.1), to give a second-order non-linear ordinary differential equation for the temperature $T(x)$, in the form

$$
F(T) T^{\prime \prime}(x)+G(T)\left[T^{\prime}(x)\right]^{2}+H(T)=0,
$$

where we have defined the functions

$$
\begin{aligned}
& F(T)=\left[T^{2}-T+\theta_{a}\right] / T^{2} \\
& G(T)=\left[\left(1-2 \theta_{a}\right) T-\theta_{a}\right] / T^{4}, \\
& H(T)=\left[\left[\mu-\beta\left(T-\theta_{a}\right)\right] e^{-1 / T}\right] / \sigma \beta
\end{aligned}
$$

A corner forms in the temperature profile at a point where the second derivative becomes singular. This is only possible in the differential equation (5.3) if the coefficient function $F(T)$ is zero at that point. Setting $F(T)=0$ and solving for $T$ gives the result (5.1).

The proof of the result (5.2) is a little more involved, and requires a local analysis of the corner region in the profile. The difficulty arises from the fact 
that $T(x)$ and its derivatives are generalised functions in the neighbourhood of the corner. We therefore consider the one-parameter family of $C^{\infty}$ functions

$$
T(x)=T_{\max }-\gamma \sqrt{\left(x-x_{c}\right)^{2}+p}, \quad p>0
$$

with the intention of eventually allowing $p \rightarrow 0$. Here, the symbol $x_{c}$ denotes the position of the corner in the profile. The function $T(x)$ and its derivatives are obtained from (5.4) and evaluated at $x=x_{c}$. The differential equation (5.3) then becomes

$$
\begin{aligned}
& -\frac{\left(1-2 T_{\max }\right) \gamma^{2}+\gamma^{3} \sqrt{p}}{T_{\max }^{2}-2 \gamma \sqrt{p} T_{\max }+\gamma^{2} p} \\
& \quad+\frac{\left[\mu-\beta\left(T_{\max }-\theta_{a}\right)+\gamma \beta \sqrt{p}\right]}{\sigma \beta} \exp \left(-\frac{1}{T_{\max }-\gamma \sqrt{p}}\right)=0 .
\end{aligned}
$$

Allowing $p \rightarrow 0$ and solving for the slope $\gamma$ gives the result (5.2). Now because of the symmetry of the profile (5.4) about the corner point $x=x_{c}$, the angle enclosed internally by the profile is $\pi-2 \arctan (\gamma)$. This concludes the proof of Theorem 2.

In Figure 5(a), we have plotted a segment of the curve (5.4) with $p=0$, and with the value of $\gamma$ estimated from (5.2). Here, the true value of $\sigma$ at the limiting case is unknown, and so the approximate value $\sigma=0.312$ obtained numerically has been used. The topmost portion of this line (labelled "maximum" on Figure 5(a)) suggests that the profile of larger amplitude in Figure 5(a) is very close to the theoretical maximum.

As anticipated from the information in Figures 3, the inclusion of a small amount of thermal diffusivity (non-zero $\alpha$ ) is expected to make a substantial qualitative difference to the results, as it removes the degeneracy of the $\alpha=0$ case. These differences are highlighted by contrasting Figure 6 with Figure 4 . In Figure 6, we display the variation in the computed eigenvalue $\sigma$ (the chemical diffusion coefficient) with the concentration amplitude $A_{C}$ of the pattern. Here, the parameter values are $\theta_{a}=0.18, \mu=0.5, \beta=1$ and $\alpha=0.001$.

One immediate qualitative difference between Figure $4(\alpha=0)$ and Figure 6 ( $\alpha=0.001$ ) is in the number of physically meaningful eigensolutions, for which the computed eigenvalue $\sigma$ is non-negative. Because of the degeneracy associated with the $\alpha=0$ case, there is an infinite spectrum of possible patterns, as was suggested by the linearised solution in Figure 3(a). However, for ambient temperature $\theta_{a}=0.18$, Figure 3(b) predicts that only the first two eigensolutions have physical meaning, and this is confirmed by the nonlinear results in Figure 6. 


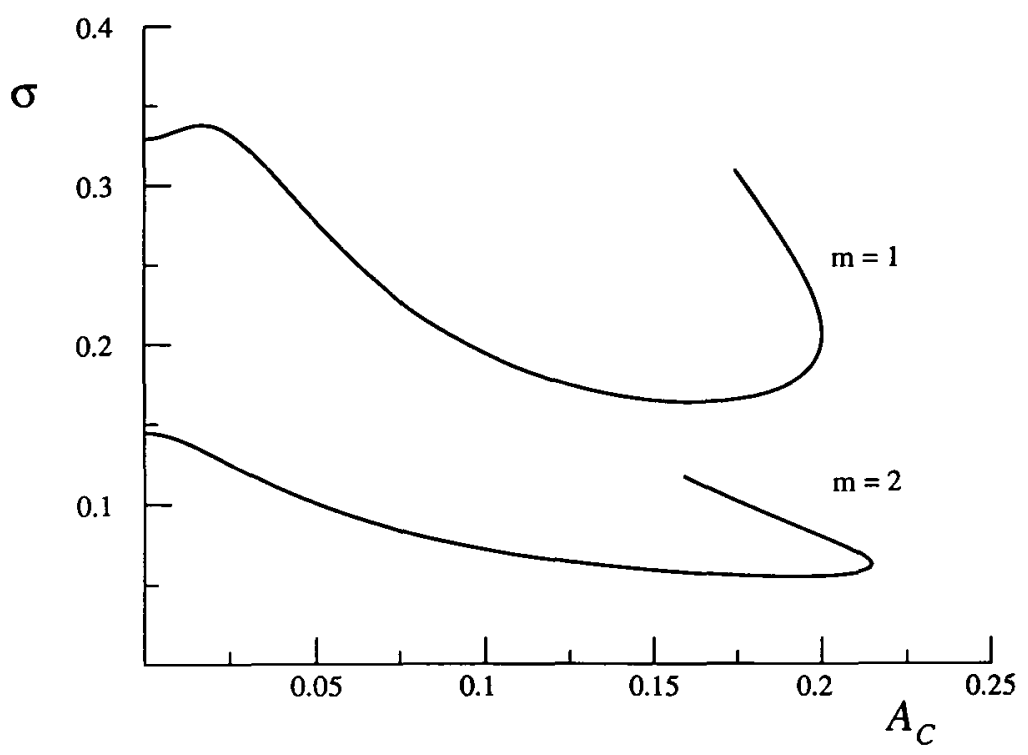

FIGURE 6. The variation of the diffusion coefficient $\sigma$ with pattern amplitude $A_{C}$, for $\theta_{a}=0.18$, $\mu=0.5, \beta=1$ and $\alpha=0.001$. Results are shown for the two eigensolutions $m=1,2$.

Another important difference between the two sets of results is that all the solutions computed in Figure 6 for $\alpha=0.001$ were found to be stable, as expected from the linearised solution and confirmed by the stability test of section 3, whereas the solutions for $\alpha=0$ in Figure 4 were seen to be unstable. In addition, the solution branches in Figure 4 for $\alpha=0$ are ultimately limited in each case by the formation of a corner singularity at some point along the profile. However, when $\alpha=0.001$, we have not detected any such limiting profile, and it was only the mounting expense in computer run-time which prevented us from continuing the solution curves in Figure 6 to larger values of $\sigma$.

Figures 4 and 6 also differ with respect to the uniqueness of solutions for a given set of physical parameters. When $\alpha=0$ as in Figure 4 , there can evidently only be a single solution profile and eigenvalue $\sigma$ for each choice of parameters. However, the results in Figure 6 for $\alpha=0.001$ reveal a genuine lack of uniqueness in the solutions, even for a pre-determined eigensolution $m$. The two curves in Figure 6 both undergo folds at some maximum value of the pattern amplitude $A_{C}$, so that specifying an amplitude for the pattern could give at least two different stable profiles. It should, however, be pointed out that the folds in these solution branches are to some extent a consequence 
(a)

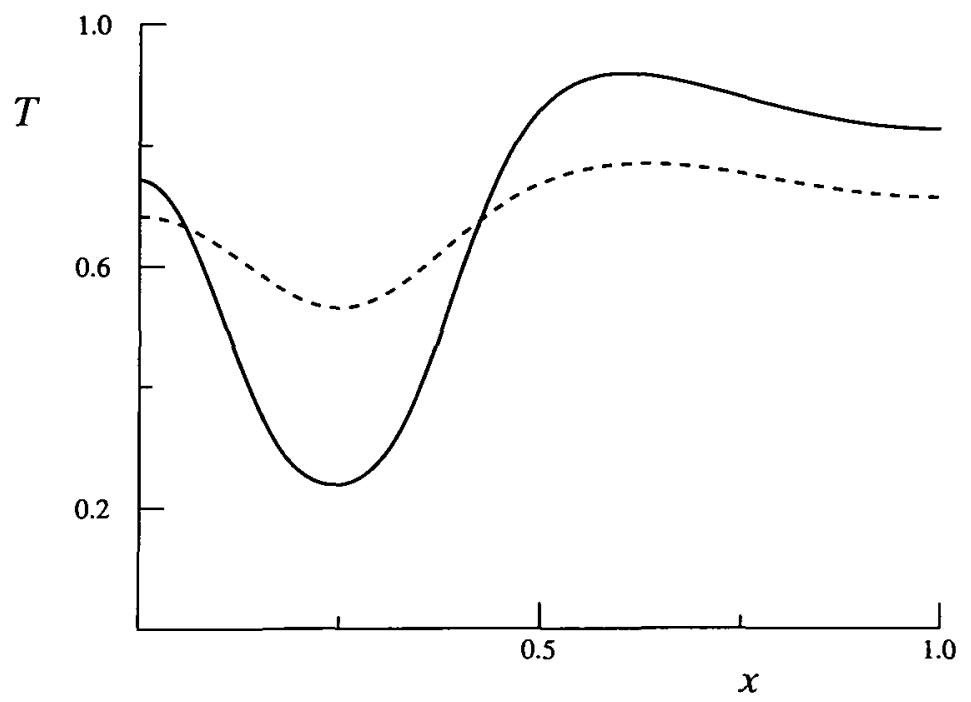

(b)

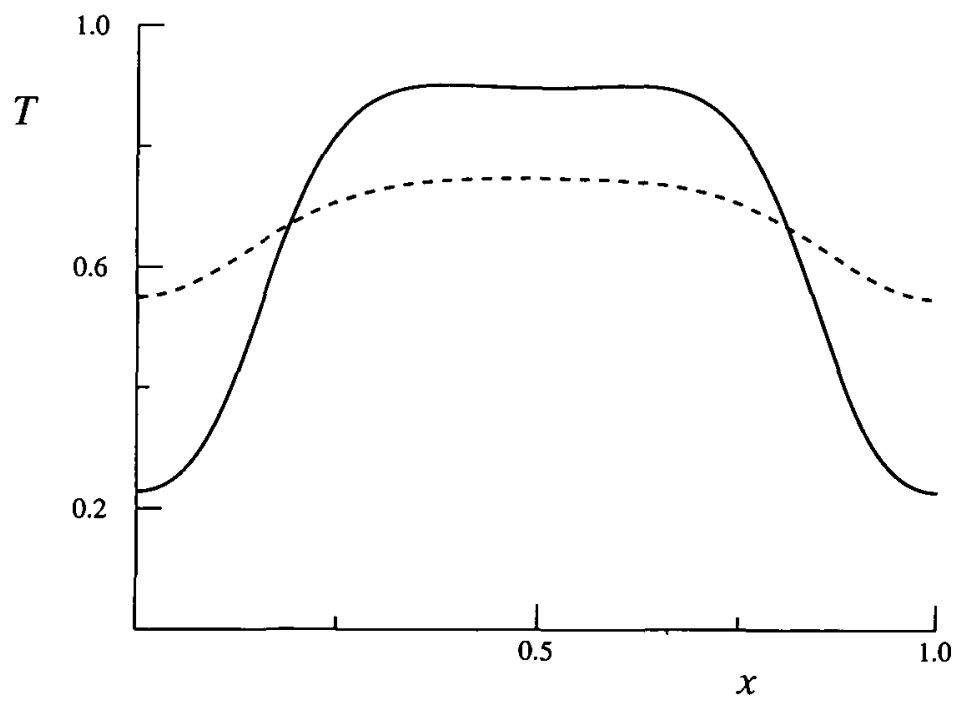

FIGURE 7. Stable temperature profiles for $\theta_{a}=0.18, \mu=0.5, \beta=1$ and $\alpha=0.001$. Results are shown for (a) The first eigensolution $m=1$, with $\sigma=0.25$. The two solutions correspond to $A_{C}=0.062596752$ (dashed line) and $A_{C}=0.19333040$ (solid line). (b) The second eigensolution $m=2$, with $\sigma=0.1$. The two solutions correspond to $A_{C}=0.050573890$ (dashed line) and $A_{C}=0.17695380$ (solid line). 


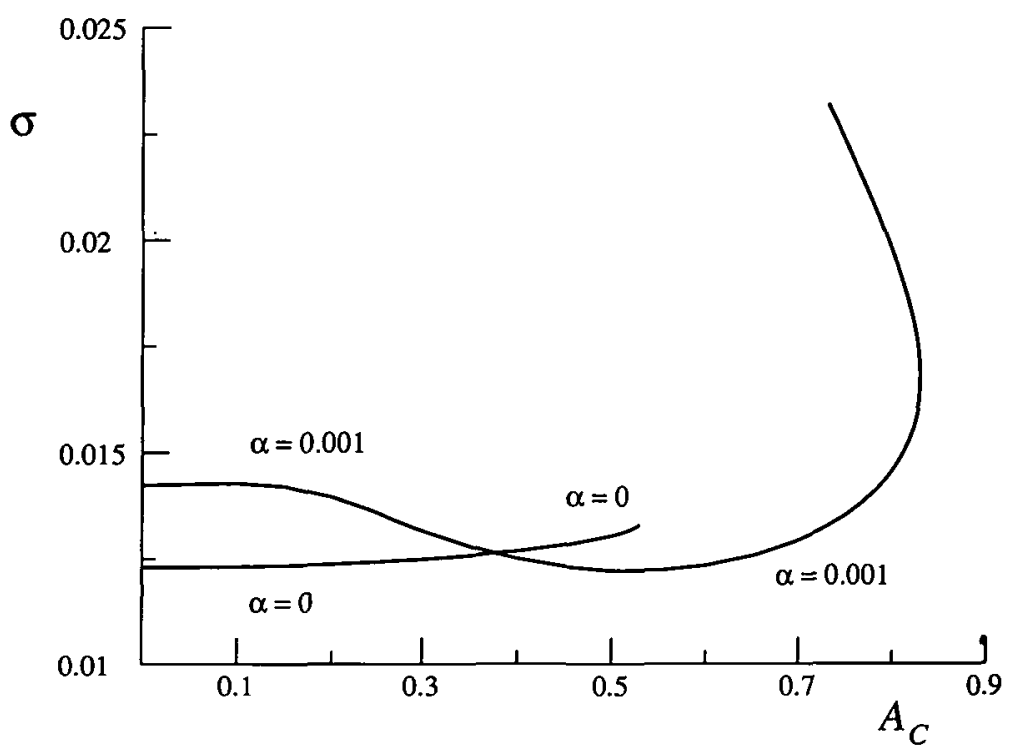

FIGURE 8. The variation of the diffusion coefficient $\sigma$ with pattern amplitude $A_{C}$, for $\theta_{a}=0.1$, $\mu=0.5$ and $\beta=1$. Results are shown for $\alpha=0$ and $\alpha=0.001$, with $m=2$.

of the definition (2.4) for pattern amplitude $A_{C}$, and an alternative definition might move the position of the fold, or even remove it entirely. Nevertheless, horizontal lines drawn through Figure 6 may intersect a given solution branch at least twice; this represents genuine non-uniqueness, since a single choice of the physical parameter $\sigma$ may result in one of at least two different stable patterns, each of different amplitude $A_{C}$. A similar fact was observed by Forbes [6] for equations governing pattern formation in the Belousov-Zhabotinskii reaction.

Some temperature profiles are displayed for this case, $\theta_{a}=0.18, \mu=0.5$, $\beta=1$ and $\alpha=0.001$, in Figures 7(a) and (b). The two solutions in Figure 7(a) both correspond to the same eigenfunction $m=1$, and to the identical value $\sigma=0.25$ of the chemical diffusion coefficient. (Notice that here, and in computing the fold singularities in Figure 6, we have employed a variant of the numerical method of Section 2, in which now the coefficient $\sigma$ is given, and the pattern amplitude $A_{C}$ computed as part of the solution.) Each of the profiles shown in Figures 7 is stable, and the presence of superharmonic oscillations is particularly evident in Figure 7(a). The two solutions in Figure 7(b) are nonunique temperature profiles for the second eigenfunction $m=2$, computed with $\sigma=0.1$. 
In Figure 8, we contrast results for the degenerate case $\alpha=0$ with results obtained for $\alpha=0.001$, for the second eigensolution $m=2$ and with parameter values $\theta_{a}=0.1, \mu=0.5$ and $\beta=1$. These values are within the region in Figure 2 enclosed by the Hopf curve, and the linearised solutions are therefore unstable. When $\alpha=0$, the computed values of the diffusion coefficient $\sigma$ do not vary much from the linearised value given by (4.6), which corresponds to pattern amplitude $A_{C}=0$. In this case, there is again a limiting value of amplitude at which the solution branch suddenly fails; at this point, a corner is formed as described in Theorem 2. When $\alpha=0.001$, however, there is again a fold singularity at a maximum value of amplitude of about $A_{C}=0.8294$, and a genuine lack of uniqueness in the possible solutions to the problem. The stability test of Section 3 confirms that the solutions in Figure 8 are unstable, as predicted by the linearised theory. No limiting profile has been detected for the branch of solutions in Figure 8 obtained with $\alpha=0.001$, and it is again only the computational expense which has prevented us from continuing this solution branch further.

The profile of the largest amplitude that could be computed accurately by our numerical method, for the $\alpha=0$ branch of solutions in Figure 8, is sketched in Figure 9. It is very close to the limiting configuration described in Theorem 2, with a corner point occurring at the maximum temperature (5.1).

Two profiles are shown in Figure 10 for the same case as in Figure 8, with $\alpha=0.001$ and pattern amplitude $A_{C}=0.73$. Both profiles are unstable, but the test of section 3 reveals that a disturbance of small amplitude to these profiles would only grow very slowly initially, and thus the patterns might persist long enough to be visible for some time in the laboratory. Notice that the ambient temperature is $\theta_{a}=0.1$ in this case, and the patterns shown thus represent very large variations in temperature across the burning surface.

\section{Conclusions}

The problem of pattern formation in an exothermic chemical reaction has been studied here in detail. A linearised analysis, valid for patterns of very small amplitude, has been presented, and the predictions are then available to guide the extensive numerical investigation of patterns of large amplitude given here.

In the linearised theory, the stability of the stationary patterns has been related to the Hopf bifurcation condition, at which a well-mixed system can undergo 
(a)

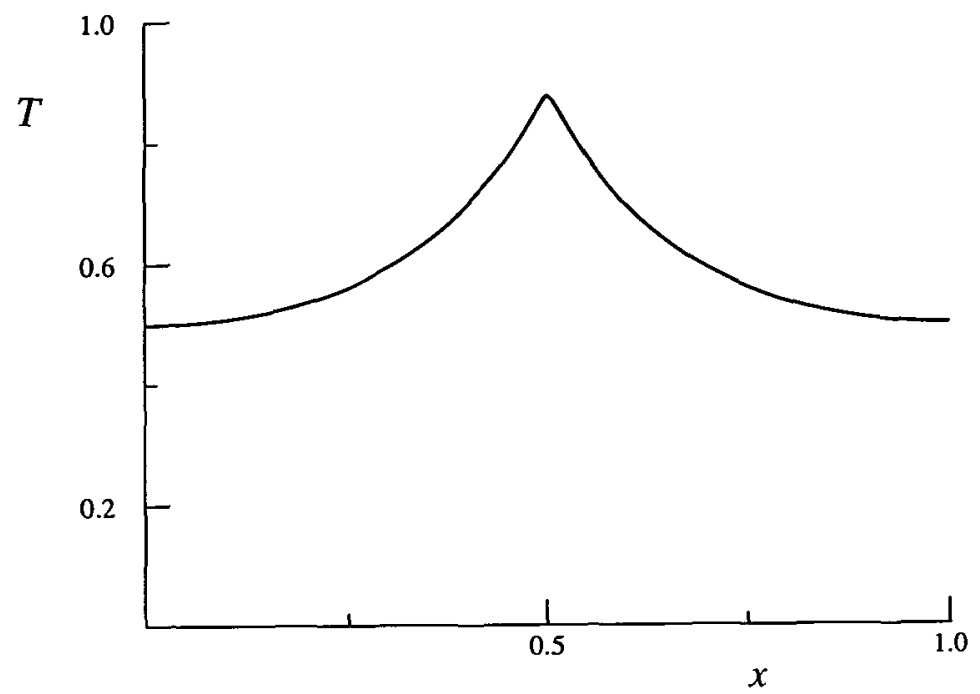

FIGURE 9. Unstable temperature profile for $\theta_{a}=0.1, \mu=0.5, \beta=1, \alpha=0$ and $m=2$. The solution has been obtained with $\sigma=0.0133\left(A_{C}=0.52977891\right)$.

(b)

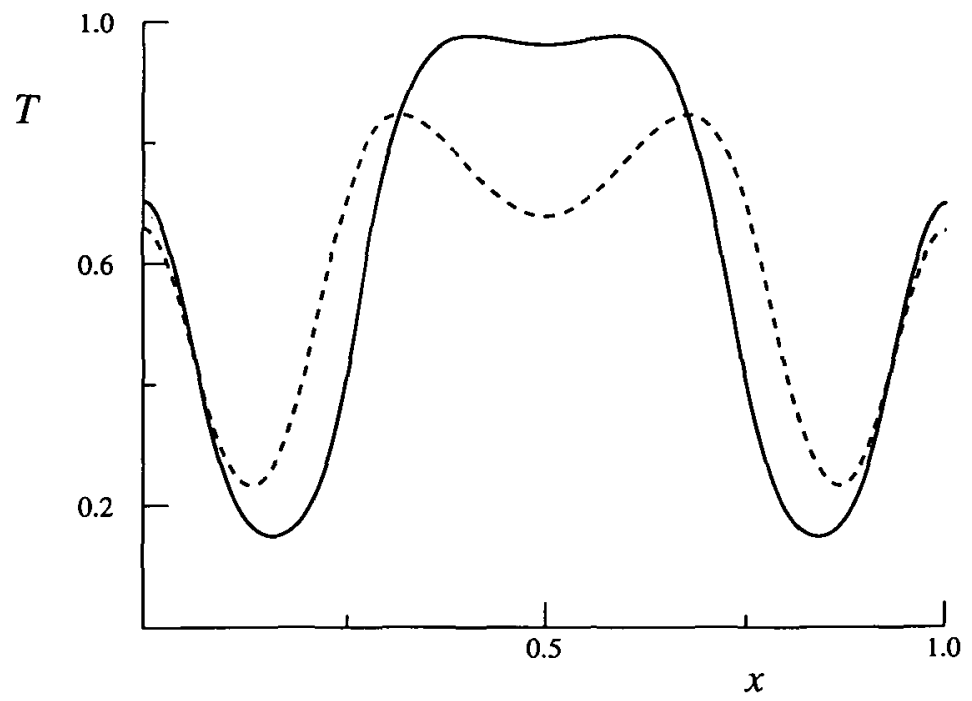

FIGURE 10. Unstable temperature profiles for $\theta_{a}=0.1, \mu=0.5, \beta=1, \alpha=0.001$ and $m=2$. The solutions have both been obtained with $A_{C}=0.73$, for $\sigma=0.013251349$ (dashed line) and $\sigma=0.023222615$ (solid line). 
periodic behaviour in time. When such behaviour is possible, the patterns are not stable, but ultimately decay to a spatially uniform state which is time-oscillatory. There is, however, a small region in parameter space outside of this oscillatory region, in which the linearised theory predicts stable patterns. Fully non-linear patterns of large amplitude also follow this general behaviour, as revealed by a stability test described in Section 3.

When there is no thermal diffusion, the patterns are unique but unstable, and they are ultimately limited by the formation of a finite-angle corner in the temperature profile, at some maximum value of temperature. In this respect, the profiles are reminiscent of the classical Stokes' wave of free-surface hydrodynamics (see Schwartz and Fenton [16]). The situation is very different, however, when thermal diffusion is included. Then the patterns may be stable, and in every case we have investigated, they are not uniquely prescribed simply by the governing physical parameters. This is obviously of physical importance, since our results show that a laboratory experiment in which all the parameters were carefully monitored could nevertheless have at least two quite different outcomes, both of which could well be stable; which one is actually obtained is presumably dependent upon the initial conditions for the reagents in the experiment.

We conclude with an observation about the patterns which the stability test of Section 3 shows to be unstable. In cases such as the two large-amplitude, unstable patterns in Figure 10, there are eigenvalues of the matrix $\mathbf{H}$ in (3.6) which have positive real parts and are thus responsible for the growth of a certain Fourier mode, and the consequent instability of the pattern. However, in most cases we have investigated, the positive real parts of these eigenvalues are extremely small, and it therefore follows that, although the affected Fourier modes must ultimately grow and destroy the pattern stability, the initial growth rate would be so small that it would not be noticed until some time later. These patterns could thus usefully be thought of as quasi-stable, and could certainly be expected to persist long enough to be observed comfortably in the laboratory in many cases. This is consistent with the experience of Forbes [6] for pattern formation in the Belousov-Zhabotinskii reaction.

\section{Acknowledgements}

This research has been done entirely whilst on a four-month sabbatical, and I am indebted to The University of Queensland for its generous support of the work, under a Special Studies Programme. I am grateful to many of my 
colleagues in the analysis and applied mathematics areas, at the University of Queensland, for their patience and willingness to advise on Section 3 on stability. A significant portion of this work was done in the School of Mathematics at the University of Leeds, and the initial problem formulation was discussed with Prof. J.H. Merkin and Dr. S.K. Scott.

\section{References}

[1] P. K. Becker and R. J. Field, "Stationary concentration patterns in the Oregonator model of the Belousov-Zhabotinskii reaction", J. Phys. Chem. 89 (1985) 118-128.

[2] J. Brindley, N. A. Jivraj, J. H. Merkin and S. K. Scott, "Stationary-state solutions for coupled reaction-diffusion and temperature-conduction equations. I. Infinite slab and cylinder with general boundary conditions", Proc. Roy. Soc. London, Ser. A 430 (1990) 459-477.

[3] W. S. Comstock and R. J. Field, "Comment on "Stationary patterns of chemical concentration in the Belousov-ZhabotinskiI reaction"', Physica D 46 (1990) 139-143.

[4] L. K. Forbes, "Limit-cycle behaviour in a model chemical reaction: the Sal'nikov thermokinetic oscillator", Proc. Roy. Soc. London, Ser. A 430 (1990) 641-651.

[5] L. K. Forbes, "Stationary patterns of chemical concentration in the Belousov-Zhabotinskii reaction", Physica D 43 (1990) 140-152.

[6] L. K. Forbes, "On stability and uniqueness of stationary one-dimensional patterns in the Belousov-Zhabotinsky reaction", Physica D 50 (1991) 42-58.

[7] B. F. Gray and M. J. Roberts, "Analysis of chemical kinetic systems over the entire parameter space. I. The Sal'nikov thermokinetic oscillator", Proc. Roy. Soc. London, Ser. A 416 (1988) 391-402.

[8] P. Gray, S. R. Kay and S. K. Scott, "Oscillations of an exothermic reaction in a closed system. I. Approximate (exponential) representation of Arrhenius temperature-dependence", Proc. Roy. Soc. London, Ser. A 416 (1988) 321-341.

[9] P. Gray and S. K. Scott, Chemical oscillations and instabilities. Non-linear chemical kinetics (Clarendon Press, Oxford, 1990).

[10] J. F. Griffiths, "The fundamentals of spontaneous ignition of gaseous hydrocarbons and related organic compounds", Adv. Chem. Phys. 64 (1986) 203-304.

[11] S. R. Kay and S. K. Scott, "Oscillations of simple exothermic reactions in a closed system. II. Exact Arrhenius kinetics”, Proc. Roy. Soc. London, Ser. A 416 (1988) 343-359.

[12] A. McNabb and L. Bass, "A diffusion-reaction model for the cellular uptake of proteinbound ligands", SIAM J. Appl. Math. 51 (1991) 124-149.

[13] J. D. Murray, "How the leopard gets its spots", Scientific American (March 1988) 62-69.

[14] S. Rica and E. Tirapegui, "Analytical description of a state dominated by spiral defects in two-dimensional extended systems", Physica D 48 (1991) 396-424.

[15] I. Y. Sal'nikov, "Contribution to the theory of the periodic homogeneous chemical reactions", h. fiz. Khim. 23 (1949) 258-272.

[16] L. W. Schwartz and J. D. Fenton, "Strongly nonlinear waves", Ann. Rev. Fluid Mech. 14 (1982) 39-60. 
[17] A. M. Turing, "The chemical basis of morphogenesis", Philos. Trans. Roy. Soc. London B 237 (1952) 37-72.

[18] J. J. Tyson, The Belousov-Zhabotinskii reaction, Lecture notes in biomathematics Vol. 10 (Springer-Verlag, Berlin, 1976).

[19] J. J. Tyson, K. A. Alexander, V. S. Manoranjan and J. D. Murray, "Spiral waves of cyclic AMP in a model of slime mold aggregation", Physica D 34 (1989) 193-207.

[20] A. T. Winfree, "Stable particle-like solutions to the nonlinear wave equations of threedimensional excitable media", SIAM Rev. 32 (1990) 1-53.

[21] A. T. Winfree, "Rotating chemical reactions", Scientific American (June 1974) 82-95.

[22] A. T. Winfree and W. Jahnke, "Three-dimensional scroll ring dynamics in the BelousovZhabotinsky reagent and in the two - variable Oregonator model", J. Phys. Chem. 92 (1989) 2823-2832. 